\title{
Research on the Modern Architecture Planning and Design Patterns under Green and Low Carbon Background
}

\author{
Liu Yanmin \\ Xiamen Institute of Technology \\ Email : lym19650077@126.com
}

\begin{abstract}
In this paper, we conduct research on the modern architecture planning and design patterns under green and low carbon background. Nowadays, the development of the national green building although there is a big progress, but in order to realize in the true sense of the green building, on the one hand, it also need to pay more attention to feature in the architectural design and operation of concept of sustainable development. Green building long-term development cannot leave the progress of science and technology and the efforts of construction industry, the green building to save resources and reduce energy consumption. This paper combines the state-of-the-art theoretical ideas to propose the modification terms for promoting the current status that is meaningful.

Keywords: Green and Low Carbon, Background, Modern, Planning and Design, Pattern.
\end{abstract}

\section{INTRODUCTION}

Green building means for human to provide a healthy and comfortable working and living, the space of activity, and to achieve the highest efficiency use of the energy, minimally affect the environment building. Nowadays, the development of the national green building although there is a big progress, but in order to realize in the true sense of the green building, on the one hand, it also need to pay more attention to feature in the architectural design and operation of concept of sustainable development, green building, which should be extended while the meaning and the connotation of its development characteristics will become more prominent [1-2].

Against China's human resources endowment and the current problems of housing consumption, we must choose resources economical development pattern based on the listed guidelines. (1) Less emissions, low pollution, reducing environment load and make full use of natural resources and the geographical conditions, solve good ventilation, ventilation, lighting, sunlight, heat, cooling and the water treatment and emissions and biochemical treatment of organic waste, reduce waste emissions, recycling is building of career. (2) Less resource-intensive and low consumption. In the input end of the housing construction to reduce resource input, reduce the consumption of resources in the process of housing consumption. (3) Green energy-saving buildings require the application of the intelligent technology support for systems and products and improve the performance of green buildings.
Green building is the embodiment of the sustainable strategy in the field of architecture, traditional buildings because of its low energy utilization efficiency in the process of construction and operation as a result of a series of environmental, health, economic and social problems. With the essentials of green building energy efficiency design three aspects, including land and the outdoor environment, the energy conservation and indoor environment and energy use, etc.

- By the implementation of the energy-saving technology in buildings to reduce building energy consumption, to ensure that the indoor environment comfort at the same time, it involves the design method that also includes the application of advanced technology.

- Improve the construction of traditional energy, conventional energy efficiency and renewable energy building applications.

- By combining the rational use of that natural environment, artificial a forestation, building orientation and layout of the reasonable arrangement, and air flow organization and so on, to achieve the effect of energy saving.

In this paper, we conduct research on the modern architecture planning and design patterns under green and low carbon background. In the following sections, we will discuss in detail. In the following figure one, we demonstrate the sample green architecture design pattern.

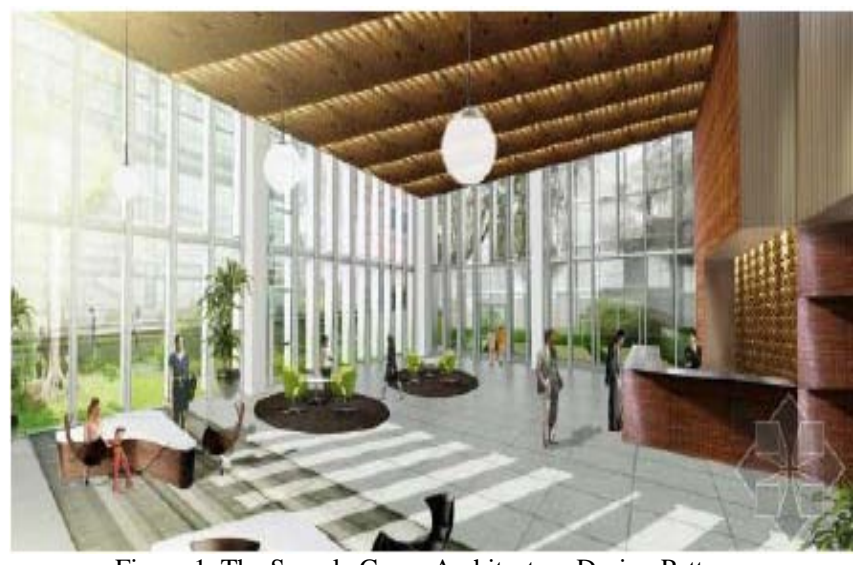

Figure 1. The Sample Green Architecture Design Pattern 


\section{The PROPOSED MethodOLOGY}

\section{A. The Green Low Carbon Background.}

With microwave energy industry, biomass, wind, solar, hydropower, nuclear power use, such as human gradually from primitive civilization to agricultural civilization and industrial civilization. And with the growing global population and economic scale, energy use brings the environment problem and its cause of the constantly been recognized, in this basic context, "carbon footprint", "low carbon economy", "low carbon technology", "low carbon development" and the series of new concepts, new policy arises at the historic moment. Advocate low carbon environmental protection in every sector of the environment and green building construction industry arises at the historic moment [3-4].

Green building long-term development cannot leave the progress of science and technology and the efforts of construction industry, the green building to save resources, reduce energy consumption, it is not damage the environment, saving environmental protection, green health, the green production efficiency, promote the sustainable development, in the development of green architecture itself. In general in our country at present, the development of the green building are mainly concentrated in the three directions: one is energy saving, the second is the environmental protection, three is to improve the construction quality, satisfy the healthy, efficient, green living conditions and people's needs.

\section{B. The Architectural Design.}

Architectural design innovation method is a scientific method and its subject is the architectural design through a series of control to the practice of architectural design innovation. Modernism architecture results produced by this kind of the practice is often can more effectively meet the needs of people is the manifestation of scientific methodology in the architectural design innovation. From a product entity, building can be regarded as a kind of engineering material, but from the design method, there is the big difference between architectural design and engineering design, which is mainly related to the natural sciences, and given priority to with logical thinking.

In view of system, science and technology and innovation is an important part of dialectics of the nature, embodies the three aspects in the architectural design is bound to be some enlightenment to the architectural design that could be roughly reflected from the listed aspects.

- Concept of science and technology. The rapid development of the science and technology as inevitably brings to the architectural design of the new design idea and thoughts. Modern architecture design must meet the requirements of science and technology view, in the current design is mainly reflected in two aspects.

- Concept of innovation. Human is always growing the nature is also growing, never stop at the same level. Therefore, the human must constantly sum up experience, find and the invention, creation and finally achieve the target of general forward.

- Systematic perspective. Modern systems theory thinks that things of widespread contact and perpetual motion is a general process, to fully grasp and basic control object, comprehensive exploration system element and element, element and system, system and environment, and the interaction between system and system and the change rule.

Active and effective control can greatly improve the success rate of architectural design innovation methods. Of architectural design innovation methods of control need to work together: first of all, the innovation process control is to meet some of the subjective and objective conditions, due to many aspects of its development, we need to choose one as the main aspects of innovation methods. System theory, from the basic point of architectural history, thought to expand and deepen the traditional architectural view, makes an area with a new perspective, as people, buildings, environment this big system of organic component. In addition, the emergence of a large number of emerging humanities also deeply affects the improvement of the architectural design method.

\section{The Architectural Planning.}

Urban planning and design work is very important to the general development of the construction of the city, and only the harmonious and reasonable space layout and design of the system can promote the development of urban construction planning work for the better.

In particular, the urban planning should be hidden under the special focus on the overall individual characteristics, because of the individual is the basic unit of the whole, and overall appeal.

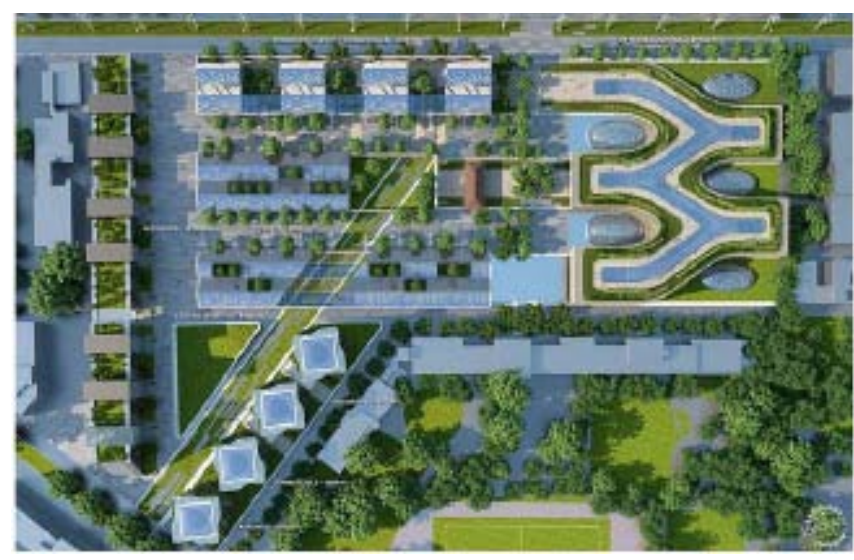

Figure 2. The Sample Architectural Planning Result

Building design is the designer of the use function of the building whole section reflects on the basis of practical, economic, beautiful some meticulous division and setting, makes the city overall planning in the aspect of urban spatial structure and performance of implementation of a reasonable.

At the same time, the design of the building mainly reflects the different characteristics of different period, makes the city building exterior effect and time to market, 
and the urban cultural environment mutual coordination, highlighting the characteristics of an era and show a different style of a city, and finally to achieve coordination between the building and city planning and development. Engineering construction design improvements are obvious, the new structure, new materials, new technology makes construction quality improved, the efficiency is greatly improved.

- The new architectural design of the pursuit of the harmony with the environment. Building construction does not damage environment as the bottom line, the pursuit of the environment coordinated goals. On the basis of absorbing the essence of the ancient architecture design concept, design pay attention to comply with the basic geographical environment, follow the geographical landscape, respect the local actual situation [5].

- When designing a building, not only consider the building itself while also considering the surrounding environmental factors, considering the role of the building in the city. With no negative effect for the bottom line, to become better in the pursuit of goals.

- Buildings are the embodiment of the social culture since ancient times, the architectural style of change means the cultural atmosphere of the basic change. New engineering design of the architectural design is not cold, it is more absorb and reflect the development of contemporary culture while the contemporary culture melts into the modern architecture makes modern architecture reflects the carrier of architectural culture.

\section{The Green Building.}

Sustainable development is to meet the needs of modern people without hurting the ability of future generations to satisfy their own needs that is the pursuit of generation and between generations is fair, a kind of coordinated development between man and nature theory and practice. The sustainable development of green building only comply with the existing legislation or policy to establish environment management goal is not enough, realize the goal of social fairness in the concept of sustainable development embedded requires construction companies to shoulder more social responsibilities. In short, the sustainable development for the basic construction industry to be responsible for environmental and social behavior to promote the development of economy while the development of the green building in addition to efforts to overcome the construction impact on the environment, to achieve the goal of fully considering the social fair.

The emergence of green building marks the traditional architectural design from the only structure of the architectural aesthetics, space utilization, form, color, structure, color and other considerations, gradually to look at from the perspective of ecological architecture, which means that the building is not only to as nonliving elements, and more is seen as the an integral part of the ecological circulation system. Under this basis, we propose the characteristics of the green building as follows.
- People-oriented, adjust measures to the local conditions, human is the core of the design of the main body are the ultimate beneficiaries of building construction. So, the root of the green building or with the person this, because only suit the needs of people's lives, from the people's health and comfortable use requirement for basic design, so the green building to effectively enhance the value of yourself.

- Comprehensive development, overall consideration: green building design is a systematic project, has the very strong comprehensive. Green building once developed as will affect the architecture education, architectural design, construction, manufacturing, basic equipment, management, decoration, energy supply and municipal infrastructure construction and other related aspects and perspectives.

- It has the complete life cycle: reduce the energy consumption is one of the most important features of the green building and from the angle of time, the characteristics of green building energy saving can be embodied in single building life cycle. Construction of environmental protection, it is not only reflected in use process, but to infiltrate into all the links from the construction to the demolition and reconstruction.

\section{CONCLUSION}

In this paper, we conduct research on the modern architecture planning and the design patterns under green and basic low carbon background. To alleviate the increasingly urgent population, resource, environment, save energy and improve the utilization rate of the resources, reduce building energy consumption, achieve architectural symbiosis with nature, harmony between human and the nature, sustainable development, improve the level of "green design" and promote the development of green energy-saving buildings in China is urgent. Under this basic requirement, this paper proposes the new and innovative perspective on the architecture planning and the design patterns to promote the later development of related applications and industries.

\section{REFERENCES}

[1] Williams J D. Architecture, Planning and Design[J]. Application Development Trends, 2002(7).

[2] Zhiqiang B A, Fang B A, Guo X, et al. Evaluation on hospital architecture planning and design by the view of hospital infection and security management[J]. Chinese Hospitals, 2006.

[3] Kitagawa Y, Hasegawa T. A Study in The LandscapeArchitecture Planning and Design Process[J]. Bulletin of the Faculty of Horticulture Minamikyushu University Natural Science Cultural \& Social Science, 1984, 14:85-96.

[4] Cai L H. Discussing on the Digital Strategies of Landscape Architecture Planning and Design[J]. Chinese Landscape Architecture, 2012.

[5] Fang H E, Amp A P, Ning Z W, et al. Integration and Dialogue The Planning and Design of the Open Space at the 6th China (Xiamen) International Garden \& Flower EXPO Park[J]. Landscape Architecture, 2007. 